Contents List available at RAZI Publishing

Matriks Sains Matematik (MSMK)

Journal Homepage: http://www.razipublishing.com/journals/matriks-sainsmatematik

https://doi.org/10.26480/msmk.01.2017.25.33

\title{
New Types of Fuzzy Interior Ideals of Ordered Semigroups Based on Fuzzy Points
}

Faiz Muhammad Khan', Nor Haniza Sarmin², Asghar Khan² and Hidayat Ullah Khan ${ }^{4 *}$

${ }^{1}$ Department of Mathematics and Statistics, University of Swat, Khyber Pakhtunkhwa, Pakistan

${ }^{2}$ Department of Mathematical Sciences, Faculty of Science Universiti Teknologi Malaysia 81310 UTM, Johor Bahru, Johor, Malaysia

${ }^{3}$ Department of Mathematics, Abdul Wali Khan University Mardan, Khyber Pakhtunkhwa, Pakistan

${ }^{4}$ Department of Mathematics, University of Malakand, Khyber Pakhtunkhwa, Pakistan *Corresponding Author email:

hidayatullak@yahoo.com

This is an open access article distributed under the Creative Commons Attribution License, which permits unrestricted use, distribution, and reproduction in any medium, provided the original work is properly cited

\section{ARTICLE DETAILS}

Article history:

Received 30 September 2016

Accepted 15 December 2016

Available online 13 January 2017

Keywords:

Fuzzy subsets; Fuzzy interior ideals; $\left(\in, \in \vee q_{k}\right)$-Fuzzy interior ideals; $\left(\in, \in \vee q_{k}\right)$-Fuzzy ideals; Semi-prime $\left(\in, \in \vee q_{k}\right)$-Fuzzy ideals; Regular (resp. left regular, right regular, intra-regular, simple and semi-simple) ordered semigroups.

\section{ABSTRACT}

Subscribing to the Zadeh's idea on fuzzy sets, many researchers strive to identify the key attributes of these sets for new finding in mathematics. In this perspective, new types of fuzzy interior ideals called $\left(\in, \in \vee q_{k}\right)$-fuzzy interior ideals of ordered semigroups are reported. Several classes of ordered semigroups such as regular ordered semigroups, intra-regular, simple and semi-simple ordered semigroups are characterized by $\left(\in, \in \vee q_{k}\right.$ )-fuzzy interior ideals and $\left(\in, \in \vee q_{k}\right)$-fuzzy ideals. We also prove that in regular (resp. intra-regular and semisimple) ordered semigroups the concept of $\left(\in, \in \vee q_{k}\right)$-fuzzy ideals and $\left(\in, \in \vee q_{k}\right)$-fuzzy interior ideals coincide. Further, we show that an ordered semigroup $S$ is simple if and only if it is $\left(\in \in \in \vee q_{k}\right)$-fuzzy simple. The characterization of intra-regular and semi-simple ordered semigroups in terms of $\left(\in, \in \vee q_{k}\right)$-fuzzy ideals and ( $\left.\in, \in \vee q_{k}\right)$-fuzzy interior ideals are provided. We define semiprime $\left(\in, \in \vee q_{k}\right)$-fuzzy ideals and prove that $S$ is left regular if and only if every $\left(\in, \in \vee q_{k}\right)$-fuzzy left ideal is semiprime and $S$ is intra-regular if and only if every ( $\left.\in, \in \vee q_{k}\right)$-fuzzy ideal is semiprime. The concept of upper/lower parts of an $\left(\in, \in \vee q_{k}\right)$-fuzzy interior ideal and some interesting results are discussed.

\section{INTRODUCTION}

Ordered semigroups arise by considering different numerical semigroups, semigroups of functions and binary relations, semigroups of subsets (or subsystems of different algebraic systems, for example ideals in rings and semigroups), etc. Every ordered semigroup is isomorphic to a certain semigroup of binary relations, considered as an ordered semigroup, where the order is set-theoretic inclusion. The classical example of a lattice-ordered semigroup is the semigroup of all binary relations on an arbitrary set. A theory of fuzzy sets on ordered semigroups can be developed. Using the idea of a quasi-coincidence of a fuzzy point with a fuzzy set, the concept of an $(\alpha, \beta)$-fuzzy interior ideal in an ordered semigroup can be developed. The idea of a quasi-coincidence of a fuzzy point with a fuzzy set, which is mentioned in a research, (Bhakat and Das, 1996a) which played a vital role to generate some different types of fuzzy subgroups (Bhakat and Das, 1992a). It is worth pointing out that Bhakat and Das gave the concepts of $(\alpha, \beta)$-fuzzy subgroups by using the belongs to relation $(\epsilon)$ and quasi-coincident with relation $(q)$ between a fuzzy point and a fuzzy subgroup, and introduced the concept of an ( $\in, \in \vee q$ )-fuzzy subgroup (Bhakat and Das, 1996a). In particular, $(\in, \in \vee q)$-fuzzy subgroup is an important and useful generalization of Rosenfeld's fuzzy subgroup. It is now natural to investigate similar type of generalizations of the existing fuzzy subsystems of other algebraic structures. With this objective in view, Davvaz introduced the concept of
( $\in, \in \vee q$ )-fuzzy sub-near-rings ( $R$-subgroups, ideals) of a near-ring and investigated some of their interesting properties (Davvaz, 2016). Jun and Song discussed general forms of fuzzy interior ideals in semigroups (Jun and Song, 2006). The concept which introduced of a generalized fuzzy biideal in semigroups and gave some properties of fuzzy bi-ideals in terms of ( $\in, \in \vee q$ )-fuzzy bi-ideals (Kazanci and Yamak). Jun et al., gave the concept of a generalized fuzzy bi-ideal in ordered semigroups and characterized regular ordered semigroups in terms of this notion (Jun et al., 2009). Generalizing the concept of $(\in, \in \vee q)$-fuzzy ideals in semigroup, Shabir et al defined $\left(\in, \in \vee q_{k}\right)$ fuzzy ideals in semigroup and characterized regular semigroups (Shabir et al., 2010). The concept of an $\left(\in, \in \vee q_{k}\right)$ )-fuzzy ideal and $\left(\in, \in \vee q_{k}\right)$-fuzzy generalized bi-ideal in ordered semigroups are given and several characterization results are discussed (Jun et al., 2012; Khan et al., 2012). Many other researchers used the idea of generalized fuzzy sets and gave several characterizations results in different branches of algebra, for example (Jun and Song, 2006; Kehayopulu, 1992; Khan et al., ; Pu and Liu., 1980 ; Shabir et al., 2010).

This paper is divided in the following sections, in section 2 , we give some basic definitions and preliminaries lemmas of interior ideals and fuzzy interior ideals of ordered semigroups. In section 3 , we give the characterizations of interior ideals in terms of $(\in, \in \vee q)$-fuzzy interior ideals and prove that in regular (resp. intra-regular and semi-simple) 
ordered semigroups the concepts of $\left(\in, \in \vee q_{k}\right)$-fuzzy interior ideals and -fuzzy ideals coincide. $\left(\in, \in \vee q_{k}\right)$ In section 5 , we introduce the concept of $\left(\in, \in \vee q_{k}\right)$-fuzzy simple ordered semigroups, we also characterize semi-simple ordered semigroups in terms of $\left(\in, \in \vee q_{k}\right)$ -fuzzy interior ideals.

\section{Preliminaries}

By an ordered semigroup (or po-semigroup) we mean a structure $(S, \cdot, \leq$ ) in which $(S, \cdot)$ is a semigroup, $(S, \leq)$ is a po set and $a \leq b \rightarrow a x \leq b x$ and $a x \leq x b$ for all $a, b, x \in S$.

For $A \subseteq S$, we denote $(A]:=\{t \in S \mid t \leq h$ for some $h \in A\}$. If $A=\{a\}$ , then we write (a] instead of $(\{a\}]$. For $A, B \subseteq S$, we denote, $A B:=\{a b \mid a \in A, b \in B\}$. In mathematics, an ordered semigroup is a

semigroup together with a partial order that is compatible with the semigroup operation. Ordered semigroups have many applications in the theory of sequential machines, formal languages, computer arithmetic's, and error-correcting codes. Ordered semigroup are mostly studied by Kehayoulu-Tesingelis.

Let $(S,, \leq)$ be an ordered semigroup. A nonempty subset $A$ of $S$ is called an interior ideal of $S$ if $A^{2} \subseteq A, S A S \subseteq A$ and $b \leq a \in A$, for $b \in S$ implies that $b \in A$.

Let $(S,, \leq)$ be an ordered semigroup. A nonempty subset $A$ of $S$ is called a left (resp. right) ideal of $S$ if

(1) If $b \leq a \in A$, for $b \in S$ then $b \in A$;

(2) $S A S \subseteq A$ (resp. $A S \subseteq A$ ).

A nonempty subset $A$ of $S$ is called an ideal if it is both a left and a right ideal of $S$.

Obviously, every ideal of an ordered semigroup $S$ is an interior ideal of $S$. We denote by $I(a)=(a \cup S a \cup a S \cup S a S$ ] (resp. $L(a)=(a \cup S a$ ] and $R(a)=(a \cup a S])$ the two-sided ideal (resp. left and right) ideal of $S$ generated by $a(a \in S)$.

An ordered semigroup $(S, \cdot, \leq)$ is called regular if for each $a \in S$ there exists $x \in S$ such that $a \leq a x a$. Equivalent Definitions: (1) $A \subseteq(A S A]$ for each $A \subseteq S$. (2) $a \in$ (aSa] for each $a \in S$ (Shabir and Khan, 2008). An ordered semigroup $S$ is called left (resp. right) regular if for each, $a \in S$ there exists $x \in S$ such that $a \leq x a^{2}$ (resp. $a \leq a^{2} x$ ), or equivalently, (1) $A \subseteq\left(A^{2} S\right.$ ] (resp. $\left.A \subseteq\left(S A^{2}\right]\right)$. (2) $a \in\left(a^{2} S\right]$ (resp.) $a \in\left(S a^{2}\right]$. An ordered semigroup $(S, \cdot, \leq)$ is called intra-regular if for each $a \in S$ there exist $x, y \in S$ such that $a \leq x a^{2} y$. Equivalent Definitions: (1) $A \subseteq\left(S A^{2} S\right.$ ] for each $A \subseteq S$. (2) $a \in\left(S a^{2} S\right.$ ] for each $a \in S$ (Shabir ${ }^{1}$ et al., 2009). An ordered semigroup $(S, \cdot, \leq)$ is called semi-simple if for each $a \in S$, there exist $x, y, z \in S$ such that $a \leq x a y a z$. Equivalent Definition: (1) $A \subseteq$ (SASAS] for each $A \subseteq S$. (2) $a \in(S a S a S$ ] for each $a \in S$ (shabir et al,.2009). An ordered semigroup $S$ is called simple if it does not contain proper ideals, that is, for any ideal $A$ of $S$, we have $A=S$ (Kehayopulu1, 1992; Kehayopulu², 1993). A non-empty subset $T$ of an ordered semigroup $S$ is called semiprime, if $a^{2} \in T$ implies $a \in T$, or equivalently, $A^{2} \subseteq T$ implies $A \subseteq T$ for all $A^{2} \subseteq S$ (Khan and Shabir, 2009).

In regular (resp. intra-regular and semi-simple ordered semigroups) the concepts of an ideal and interior ideal coincide.

A fuzzy subset $F$ from a universe $X$ is a function from $X$ into unit closed interval $[0,1]$ of real numbers, i.e., $F: X \rightarrow[0,1]$.

For any two fuzzy subsets $F$ and $G$ of $S, F \leq G$ means that $F(x) \leq G(x)$, for all $x \in S$. The symbols $F \wedge G$ and $F \vee G$ will mean the following fuzzy subsets: $(\forall x \in S)(F \vee G: S \rightarrow[0,1], x \mapsto(F \vee G)(x)=\max \{F(x), G(x)\})$.

Let $a \in S$ and $\phi \neq A \subseteq S$, we denote $A_{a}$ by

$A_{a}=\{(y, z) \in S \times S \mid a \leq y z\}$.

Let $F$ and $G$ be any two fuzzy subsets of an ordered semigroup $S$, the product $F \circ G$ of $F$ and $G$ is defined by:

$F \circ G: S \rightarrow[0,1], a \mapsto(F \circ G)(a)=\left\{\begin{array}{cc}\bigvee_{(y, z) \in A_{a}} \min \{F(y), G(z)\} & \text { if } A_{a} \neq \phi \\ 0 & \text { if } A_{a}=\phi\end{array}\right.$

Definition 2.1 A fuzzy subset $F$ of $S$, is called a fuzzy interior ideal of $S$, if

(1) $(\forall x, y \in S)(x \leq y \rightarrow F(x) \geq F(y)$;

(2) $(\forall x, y \in S)(F(x y) \geq \min \{F(x), F(y)\}$

(3) $(\forall x, a, y \in S)(F(x a y) \geq F(a))$.

Definition 2.2A fuzzy subset $F$ of $S$ is called a fuzzy left (resp. right) ideal of $S$, if 2

(1) $(\forall x, y \in S)(x \leq y \rightarrow F(x) \geq F(y))$ and

(2) $(\forall x, y \in S)(F(x y) \geq F(y)$ (resp. $F(x y) \geq F(x))$.

In regular (resp. intra-regular and semi-simple ordered semigroups) the concepts of a fuzzy ideal and fuzzy interior ideal coincide.

Definition 2.3 A fuzzy subset $F$ of $S$ is called semiprime if $F(x) \geq F\left(x^{2}\right)$ for all $x \in S$.

Let $S$ be an ordered semigroup and $F$ a fuzzy subset of $S$, then for all $t \in(0,1]$, the set

is called a level set of $F$

$$
U(F ; t):=\{x \in S \mid F(x) \geq t\},
$$

In the following we characterize fuzzy interior ideals of ordered semigroups in terms of level interior ideals.

Theorem 2.4 A fuzzy subset $F$ of an ordered semigroup $S$ is a fuzzy interior ideal of $S$ if and only if for all $t \in(0,1]$, the set $U(F ; t)(\neq \phi)$ is an interior ideal of $S$.

Let $(S,, \leq)$ be an ordered semi-group and $\phi \neq A \subseteq S$. Then the characteristic function $F_{A}$ of $A$ is defined by

$$
F_{A}: S \rightarrow[0,1], x \mapsto F_{A}(x):=\left\{\begin{array}{l}
1 \text { if } x \in A, \\
0 \text { if } x \notin A .
\end{array}\right.
$$

Clearly, a nonempty subset $A$ of $S$ is an interior ideal of $S$ if and only if the characteristic function $F_{A}$ of $A$ is a fuzzy interior ideal of $S$.

\section{3. $\left(\in, \in \vee q_{k}\right)$-fuzzy interior ideals}

In what follows, let $S$ denote an ordered semigroup unless otherwise specified. In this section, we define a more generalized form of $(\alpha, \beta)$

-fuzzy interior ideals of an ordered semigroups $S$ and introduced $\left(\in, \in \vee q_{k}\right)$-fuzzy interior ideals of $S$ where $k$ is an arbitrary element of $[0,1)$ unless otherwise stated. A fuzzy subset $F$ in $S$ of the form

$$
F: S \rightarrow[0,1], \quad y \mapsto \begin{cases}t \in(0,1] & \text { if } y=x, \\ 0 & \text { if } y \neq x,\end{cases}
$$

is said to be a fuzzy point with support $X$ and value $t$ and is denoted by $[x ; t]$. For a fuzzy point $[x ; t]$ is said to belong to (resp. quasicoincident with) a fuzzy set $F$, written as $[x ; t] \in F$ (resp. $[x ; t] q F$ ) if $F(x) \geq t$ ( resp. $F(x)+t \geq 1)$. If $[x ; t] \in F$ or $[x ; t] q F$, then we write $[x ; t] q F$. The symbol $\overline{\in \vee q}$ means $\in \vee q$ does not hold. Generalizing the concept of $[x ; t] q F$, in semigroups, defined $[x ; t] q_{k} F$, as 
$F(x)+t+k>1$, where $k \in[0,1)$ (Shabir et al., 2010).

(1) $(\forall x, y \in S)(\forall t \in(0,1])\left(x \leq y,[y ; t] \in F \rightarrow[x ; t] \in \vee q_{k} F\right)$,

(2)

$(\forall x, y \in S)(\forall t, r \in(0,1])\left([x ; r] \in F,[y ; t] \in F \rightarrow[x y ; r \wedge t] \in \vee q_{k} F\right)$,

(3) $(\forall x, a, z \in S)(\forall t \in(0,1])\left([a ; t] \in F \rightarrow[x a z ; t] \in \vee q_{k} F\right)$.

Theorem 3.2 Let $A$ be an interior ideal of $S$ and $F$ a fuzzy subset in $S$ defined by:

$$
F(x)=\left\{\begin{array}{l}
\geq \frac{1-k}{2} \text { if } x \in A, \\
0 \quad \text { otherwise }
\end{array}\right.
$$

Then

(1) $F$ is a $(q, \in \vee q k)$-fuzzy interior ideal of $S$.

(2) $F$ is an $(\in, \in \vee q k)$-fuzzy interior ideal of $S$.

Proof (1) Let $x, y \in S, x \leq y$ and $t \in(0,1]$ be such that $[y ; t] \mathrm{q}$

$F$. Then $y \in A, F(y)+t>1$. Since $A$ is an interior ideal of $S$ and $x \leq y \in A$, we have $x \in A$. Thus $F(x) \geq \frac{1-k}{2}$. If $t \leq \frac{1-k}{2}$, then $F(x) \geq t \quad$ and $\quad$ so $[x ; t] \in F$.If $\quad t>\frac{1-k}{2}, \quad$ then $F(x)+t+k>\frac{1-k}{2}+\frac{1-k}{2}+k=1$ and so $[x ; t] \mathrm{q}{ }_{k} F$. Therefore $[x ; t] \in \vee \mathrm{q}$ ${ }_{k} F$.

Let $x, y \in S$ and $t, r \in(0,1]$ be such that $[x ; t] \mathrm{q} F$. and $[y ; r] \mathrm{q} F$. Then $x, y \in A$, so $F(x)+t>1$ and $F(y)+r>1$. Since $A$ is an interior ideal of $S$, we have $x y \in A$. Thus $F(x y) \geq \frac{1-k}{2}$. If $t \wedge r>\frac{1-k}{2}$, then $F(x y)+t \wedge r+k>\frac{1-k}{2}+\frac{1-k}{2}+k=1$ and so $[x y ; t \wedge r] \mathrm{q}_{k} F$. If $t \wedge r \leq \frac{1-k}{2}, \quad$ then $\quad F(x y) \geq t \wedge r \quad$ and $\quad$ so $\quad[x y ; t \wedge r] \in F$. Therefore, $[x y ; t \wedge r] \in \vee \mathrm{q}_{k} F$.

Let $x, a, z \in S$ and $t \in(0,1]$ be such that $[a ; t] q F$ Then $a \in A$, $F(a)+t>1$. Since $A$ is an interior ideal of $S$, we have $x a z \in A$. Thus, $F(x a z) \geq \frac{1-k}{2}$. If $t>\frac{1-k}{2}$, then $F(x a z)+t+k>\frac{1-k}{2}+\frac{1-k}{2}+k=1$ and so $[x a z ; t] \mathrm{q}_{k} F$. If $t \leq \frac{1-k}{2}$, then $F(x a z) \geq t$, and so $[x a z ; t] \in F$. Therefore, $[x a z ; t] \in \vee \mathrm{q}_{k} F$.

(2) Let $x, y \in S, x \leq y$ and $t \in(0,1]$ be such that $[y ; t] \in F$. Then $F(y) \geq t$ and $y \in A$. Since $A$ is an interior ideal of $S$ and $x \leq y \in A$, we have $x \in A$. Thus $F(x) \geq \frac{1-k}{2}$. If $t \leq \frac{1-k}{2}$, then $F(x) \geq t \quad$ and $\quad$ so $[x ; t] \in F . \quad$ If $\quad t>\frac{1-k}{2}, \quad$ then $F(x)+t+k>\frac{1-k}{2}+\frac{1-k}{2}+k=1 \quad$ and $\quad$ so $\quad[x ; t] \mathrm{q}_{k} F$. Therefore $[x ; t] \in \vee \mathrm{q}_{k} F$.

Let $x, y \in S$ and $t, r \in(0,1]$ be such that $[x ; t] \in F$ and $[y ; r] \in F$. Then $x, y \in A$. Since $A$ is an interior ideal of $S$, we have $x y \in A$. Thus $F(x y) \geq \frac{1-k}{2}$. If $t \wedge r>\frac{1-k}{2}$, then $F(x y)+t \wedge r+k>\frac{1-k}{2}+\frac{1-k}{2}+k=1$ and so $[x y ; t \wedge r]$ $\mathrm{q}_{k} F . \quad$ If $t \wedge r \leq \frac{1-k}{2}$, then $F(x y) \geq t \wedge r$ and so $[x y ; t \wedge r] \in F$. Therefore $[x y ; t \wedge r] \in \vee \mathrm{q}_{k} F$. Let $x, a, z \in S$ and $t \in(0,1]$ be such that $[a ; t] \in F$. Then $a \in A$. Since $A$ is an interior ideal of $S$, we have, $x a z \in A$. Thus, $F(x a z) \geq \frac{1-k}{2}$. If $\quad t>\frac{1-k}{2}, \quad$ then $F(x a z)+t+k>\frac{1-k}{2}+\frac{1-k}{2}+k=1$ and so [ $x a z ; t$ ]q $F$.
If $t \leq \frac{1-k}{2}$, then $F(x a z) \geq t$ and so $[x a z ; t] \in F$. Thus, $[x a z ; t] \in \vee \mathrm{q}_{k} F$. Consequently, $F$ is an $(\in, \in \vee \mathrm{qk})$-fuzzy interior ideal of $S$

If we take $k=0$ in Theorem 3.2, then we get the following corollary:

Corollary 3.3 (Khan and Shabir., 2009) Let $A$ be an interior ideal of $S$ and $S$ a fuzzy subset in $S$ defined by:

Then

(1) $S$ is a (q, $\in \vee$ q)-fuzzy interior -ideal of $S$.

(2) $F$ is an $(\in, \in \vee$ q)-fuzzy interior ideal of $S$.

Theorem 3.4 A fuzzy subset $F$ of $S$ is an $\left(\in, \in \vee \mathrm{q}_{k}\right)$-fuzzy interior ideal of $S$ if and only if

(1) $(\forall x, y \in S)\left(x \leq y \rightarrow F(x) \geq F(y) \wedge \frac{1-k}{2}\right)$,

(2) $(\forall x, y \in S)\left(F(x y) \geq F(x) \wedge F(y) \wedge \frac{1-k}{2}\right)$,

(3) $(\forall x, a, z \in S)\left(F(x a z) \geq F(a) \wedge \frac{1-k}{2}\right)$.

Proof Let $F$ be an $\left(\in, \in \vee \mathrm{q}_{k}\right)$-fuzzy interior ideal of $S$. On the contrary assume that, there exist $x, y \in S, x \leq y$ such that $F(x)<F(y) \wedge \frac{1-k}{2}$. Choose $t \in(0,1]$ such that $F(x)<t \leq F(y) \wedge \frac{1-k}{2}$. Then $[y ; t] \in F$, but $F(x)<t$ and $F(x)+t+k<\frac{1-k}{2}+\frac{1-k}{2}+k=1$, so $[x ; t] \overline{\in \vee q_{k}} F$, which is a contradiction. Hence $F(x) \geq F(y) \wedge \frac{1-k}{2}$ for all $x, y \in S$ with $x \leq y$.

If there exist $x, y \in S$ such that $F(x y)<F(x) \wedge F(y) \wedge \frac{1-k}{2}$. Choose $t \in(0,1]$ such that $F(x y)<t \leq F(x) \wedge F(y) \wedge \frac{1-k}{2}$. Then $[x ; t] \in F,[y ; t] \in F \quad$ but $\quad F(x y)<t \quad$ and $F(x y)+t+k<\frac{1-k}{2}+\frac{1-k}{2}+k=1$, so $\quad[x y ; t] \bar{q}_{k} F$. Thus, $\quad[x y ; t] \overline{\in \vee q_{k}} F$, which is a contradiction. Therefore, $F(x y) \geq F(x) \wedge F(y) \wedge \frac{1-k}{2}$ for all $x, y \in S$

Now if there exist $x, a, z \in S$ such that $F(x a z)<F(a) \wedge \frac{1-k}{2}$. Then, for some $t \in(0,1]$ such that $F(x a z)<t \leq F(a) \wedge \frac{1-k}{2}$, we have, $[a ; t] \in F$ but $F(x a z)<t$ and $F(x a z)+t+k<\frac{1-k}{2}+\frac{1-k}{2}+k=1$, so $[x a z ; t] \bar{q}_{k} F$ . Thus, $[x a z ; t] \overline{\in \vee q_{k}} F$, which is a contradiction. Therefore $F(x a z) \geq F(a) \wedge \frac{1-k}{2}$ for all $x, a, z \in S$.

Conversely, let $[y ; t] \in F$ for some $t \in(0,1]$. Then $F(y) \geq t$. Now, $F(x) \geq F(y) \wedge \frac{1-k}{2} \geq t \wedge \frac{1-k}{2}$. If $t>\frac{1-k}{2}$, then $F(x) \geq \frac{1-k}{2}$ and $F(x)+t+k>\frac{1-k}{2}+\frac{1-k}{2}+k=1$, it follows that $[x ; t] \mathrm{q}_{k} F$. If $t \leq \frac{1-k}{2}$, then $F(x) \geq t$ and so $[x ; t] \in F$. Thus, $[x ; t] \in \vee q_{k} F$.

Let $[x ; t] \in F$ and $[y ; r] \in F$, then $F(x) \geq t$ and $F(y) \geq r$. Thus $\quad F(x y) \geq F(x) \wedge F(y) \wedge \frac{1-k}{2} \geq t \wedge r \wedge \frac{1-k}{2}$. If $t \wedge r>\frac{1-k}{2}, \quad$ then $\quad F(x y) \geq \frac{1-k}{2} \quad$ and $F(x y)+t \wedge r+k>\frac{1-k}{2}+\frac{1-k}{2}+k=1$ and so $[x y ; t \wedge r] \mathrm{q}_{k} F$. If $t \wedge r \leq \frac{1-k}{2}$, then $F(x y) \geq t \wedge r$ and hence, $[x y ; t \wedge r] \in F$. Thus $[x y ; t \wedge r] \in \vee \mathrm{q}_{k} F$. Now let $[a ; t] \in F$, then $F(a) \geq t$ and we have, $F(x a z) \geq F(a) \wedge \frac{1-k}{2} \geq t \wedge \frac{1-k}{2}$. If $t>\frac{1-k}{2}$, then $F(x a z) \geq \frac{1-k}{2} \quad$ and $\quad F(x a z)+t+k>\frac{1-k}{2}+\frac{1-k}{2}+k=1$ and so $[x a z ; t] \mathrm{q}_{k} F$. If $t \leq \frac{1-k}{2}$, then $F(x a z) \geq t$ and hence, $[x a z ; t] \in F$. Thus $[x a z ; t] \in \vee \mathrm{q}_{k} F$ and consequently, $F$ 
is an $\left(\in, \in \vee \mathrm{q}_{k}\right)$-fuzzy interior ideal of $S$.

If we take $k=0$ in Theorem 3.4, we have the following corollary:

Corollary 3.5 (Khan and Shabir, 2009) Let $F$ be a fuzzy subset of $S$. Then $F$ is an ( $\in, \in \vee$ q)-fuzzy interior ideal of $S$ if and only if

(1) $(\forall x, y \in S)(x \leq y \rightarrow F(x) \geq F(y) \wedge 0.5)$,

(2) $(\forall x, y \in S)(F(x y) \geq F(x) \wedge F(y) \wedge 0.5)$,

(3) $(\forall x, a, z \in S)(F(x a z) \geq F(a) \wedge 0.5)$.

Theorem 3.6 A fuzzy subset $F$ of $S$ is an $\left(\in, \in \vee q_{k}\right)$-fuzzy interior ideal of $S$ if and only if $U(F ; t)(\neq \phi)$ is an interior ideal of $S$ for all $t \in\left(0, \frac{1-k}{2}\right]$.

Proof Suppose that $F$ is an $\left(\epsilon, \in \vee q_{k}\right)$-fuzzy interior ideal of $S$ and let $x, y \in S$ be such that $x, y \in U(F ; t)$ for some $t \in\left(0, \frac{1-k}{2}\right]$. Then $F(x) \geq t$ and $F(y) \geq t$ and by hypothesis

$$
\begin{aligned}
F(x y) & \geq F(x) \wedge F(y) \wedge \frac{1-k}{2} \\
& \geq t \wedge t \wedge \frac{1-k}{2}=t .
\end{aligned}
$$

Hence $x y \in U(F ; t)$. Let $x, a, y \in S^{2}$ be such that $a \in U(F ; t)$ for some $t \in\left(0, \frac{1-k}{2}\right]$. Then $F(a) \geq t$ and by hypothesis

$$
\begin{aligned}
F(x a y) & \geq F(a) \wedge \frac{1-k}{2} \\
& \geq t \wedge \frac{1-k}{2}=t .
\end{aligned}
$$

Hence $x a y \in U(F ; t)$.

Now let $x, y \in S, x \leq y$ be such that $y \in U(F ; t)$ for some $t \in\left(0, \frac{1-k}{2}\right]$. Then $F(y) \geq t$ and by hypothesis

Hence $x \in U(F ; t)$.

$$
\begin{aligned}
F(x) & \geq F(y) \wedge \frac{1-k}{2} \\
& \geq t \wedge \frac{1-k}{2}=t .
\end{aligned}
$$

Conversely, assume that $U(F ; t)(\neq \phi)$ is an interior ideal of $S$ for all $t \in\left(0, \frac{1-k}{2}\right]$. If there exist $x, y \in S$ such that $F(x y)<F(x) \wedge F(y) \wedge \frac{1-k}{2}$. Then choose $t \in\left(0, \frac{1-k}{2}\right]$ such that $F(x y)<t \leq F(x) \wedge F(y) \wedge \frac{1-k}{2}$. Thus $x, y \in U(F ; t) \quad$ but $x y \notin U(F ; t), \quad$ a contradiction. Hence $F(x y) \geq F(x) \wedge F(y) \wedge \frac{1-k}{2}$ for all $x, y \in S$ and $k \in[0,1)$. If there exist $x, a, y \in S$ such that $F(x a y)<F(a) \wedge \frac{1-k}{2}$. Then choose $t \in\left(0, \frac{1-k}{2}\right]$ such that $F(x a y)<t \leq F(a) \wedge \frac{1-k}{2}$. Thus $a \in U(F ; t)$ but $x a y \notin U(F ; t)$, a contradiction. Hence $F(x a y) \geq F(a) \wedge \frac{1-k}{2}$ for all $x, a, y \in S$ and $k \in[0,1)$

Let $x, y \in S$ be such that $F(x)<F(y) \wedge \frac{1-k}{2}$. Choose $r \in\left(0, \frac{1-k}{2}\right]$ such that $F(x)<r \leq F(y) \wedge \frac{1-k}{2}$ then $F(y) \geq r$ implies that $[y ; r] \in F$ but $[x ; r] \bar{\in} F$. Now $F(x)+r+k<\frac{1-k}{2}+\frac{1-k}{2}+k=1$, which implies that $[x ; r] \overline{q_{k}} F$, contradiction. Hence $F(x) \geq F(y) \wedge \frac{1-k}{2}$. Therefore $F$ is an $\left(\in, \in \vee q_{k}\right)$-fuzzy interior ideal of $S$.

Example 3.7 Consider the ordered semigroup $S=\{a, b, c, d\}$

\begin{tabular}{|c|c|c|c|c|}
\hline$\cdot$ & $a$ & $b$ & $c$ & $d$ \\
\hline$a$ & $a$ & $a$ & $a$ & $a$ \\
\hline$b$ & $a$ & $a$ & $a$ & $a$ \\
\hline$c$ & $a$ & $a$ & $b$ & $a$ \\
\hline$d$ & $a$ & $a$ & $b$ & $b$ \\
\hline
\end{tabular}

$\leq:=\{(a, a),(b, b),(c, c),(d, d),(a, b)\}$.

Then $\{a\},\{a, b\},\{a, c\},\{a, d\},\{a, b, c\},\{a, c, d\}$ and $\{a, b, c, d\}$ are interior ideals of $S$. Define a fuzzy subset $F$ of $S$ as follows:

$$
F: S \rightarrow[0,1] \mid x \mapsto F(x)=\left\{\begin{array}{l}
0.7 \text { if } x=a, \\
0.6 \text { if } x=d, \\
0.3 \text { if } x=c, \\
0.2 \text { if } x=b .
\end{array}\right.
$$

Then

$$
U(F ; t)=\left\{\begin{array}{cc}
S & \text { if } 0<t \leq 0.2, \\
\{a, c, d\} & \text { if } 0.2<t \leq 0.3, \\
\{a, d\} & \text { if } 0.3<t \leq 0.6, \\
\phi & \text { if } 0.7<t \leq 1
\end{array}\right.
$$

Then, by Theorem 3.6, $F$ is an $\left(\in, \in \vee q_{k}\right)$-fuzzy interior ideal of $S$ for $t \in\left(0, \frac{1-k}{2}\right]$ with $k=0.4$.

Proposition 3.8 If $F$ is a nonzero $\left(\in, \in \vee q_{k}\right)$-fuzzy interior ideal of $S$. Then the set $F_{0}=\{x \in S \mid F(x)>0\}$ is an interior ideal of $S$.

Proof Let $F$ be an ( $\in, \in \vee \mathrm{q}_{k}$ )-fuzzy interior ideal of $S$. Let $x, y \in S, x \leq y$ and $y \in F_{0}$. Then, $F(y)>0$. Since $F$ is an $(\in, \in \vee$ $\mathrm{q}_{k}$ )-fuzzy interior ideal of $S$, we have

$$
F(x) \geq F(y) \wedge \frac{1-k}{2}>0, \text { because } F(y)>0 .
$$

Thus $F(x)>0$ and so $x \in F_{0}$. Let $x, y \in F_{0}$. Then, $F(x)>0$ and $F(y)>0$. Now,

$$
F(x y) \geq F(x) \wedge F(y) \wedge \frac{1-k}{2}>0
$$

Thus $x y \in F_{0}$. For $a \in F_{0}$ we have

$$
F(x a z) \geq F(a) \wedge \frac{1-k}{2}>0
$$

and so $x a z \in F_{0}$, consequently $F_{0}$ is an interior ideal of $S$.

Lemma 3.9 A non-empty subset $A$ of $S$ is an interior ideal if and only if the characteristic function $F_{A}$ of $A$ is an ( $\in, \in \vee \mathrm{q}_{k}$ )-fuzzy interior ideal of $S$.

Proof The proof is obvious.

Proposition 3.10 Every ( $\in, \in \vee q_{k}$ ) -fuzzy ideal of $S$ is an ( $\in, \in \vee q_{k}$

)-fuzzy interior ideal of $S$.

Proof The proof is straightforward.

The converse of Proposition 3.10 is not true, in general.

Example 3.11 Consider the ordered semigroup $S=\{0, a, b, c\}$ with the following multiplication table and order relation

$$
\begin{array}{|l|l|l|l|l|}
\hline \cdot & 0 & a & b & c \\
\hline 0 & 0 & 0 & 0 & 0 \\
\hline a & 0 & 0 & 0 & 0 \\
\hline b & 0 & 0 & 0 & a \\
\hline c & 0 & 0 & a & b \\
\hline
\end{array}
$$


$\leq:=\{(0,0),(a, a),(b, b),(c, c),(0, a)\}$

Let $F: S \rightarrow[0,1]$ be a fuzzy subset defined by:

$F(0)=0.7, \quad F(a)=0.2, \quad F(b)=0.6, \quad F(c)=0$

Then $F$ is an $\left(\in, \in \vee q_{k}\right)$-fuzzy interior ideal of $S$ for all $k=0.4$

, but $F$ is not an ( $\in, \in \vee q_{k}$ )-fuzzy ideal of $S$. Because $F(x a z)=F(0)=0.7>0.3 \geq F(a) \wedge \frac{1-k}{2}$. Also if $\quad x y=0$, then $F(x y)=F(0)=0.7>0.3 \geq F(x) \wedge F(y) \wedge \frac{1-k}{2}$. If $x y=a$, then $F(x y)=F(a)=0.2>0=F(x) \wedge F(y) \wedge \frac{1-k}{2}$ and if $x y=b$, then $F(x y)=F(b)=0.6>0=F(x) \wedge F(y) \wedge \frac{1-k}{2}$ for every $x, z \in S$. Obviously for every $x, y \in S$, such that $x \leq y$ we have $F(x) \geq F(y) \wedge \frac{1-k}{2}$. But $F(b . c)=F(a)=0.2<0.3=F(b) \wedge \frac{1-k}{2}$. Hence $F$ is not an $\left(\in, \in \vee q_{k}\right)$ fuzzy right ideal. Thus $F$ is not an $\left(\in, \in \vee q_{k}\right)$-fuzzy ideal of $S$.

Proposition 2.12 Let $S$ be a regular ordered semigroup, then every $\left(\in, \in \vee q_{k}\right)$-fuzzy interior ideal is an ( $\left.\in, \in \vee q_{k}\right)$-fuzzy ideal of $S$.

Proof Let $F$ be an ( $\in, \in \vee q_{k}$ )-fuzzy interior ideal and $a, b \in S$.

Then there exists $x \in S$ such that $a \leq a x a$, hence we have $F(a b) \geq F((a x a) b) \wedge \frac{1-k}{2}$ (because $F$ is an $\left(\in, \in \vee q_{k}\right)$-fuzzy interior ideal)

$$
\begin{aligned}
& =F((a x) a b) \wedge \frac{1-k}{2} \\
& \geq\left(F(a) \wedge \frac{1-k}{2}\right) \wedge \frac{1-k}{2} \text { (because } F \text { is an }\left(\in, \in \vee q_{k}\right) \text { - fuzzy interior ideal) } \\
& =F(a) \wedge \frac{1-k}{2} .
\end{aligned}
$$

Similarly, we can show that $F(a b) \geq F(b) \wedge \frac{1-k}{2}$ for every $a, b \in S$. Thus $F$ is an ( $\in, \in \vee q_{k}$ ) -fuzzy ideal of $S$.

From Propositions 3.10 and 3.12, we have the following corollary:

Corollary 3.13 In regular ordered semigroups, the concepts of an ( $\in, \in \vee q_{k}$ )-fuzzy ideals and ( $\in, \in \vee q_{k}$ )-fuzzy interior ideals coincide.

Proposition 3.14 Let $S$ be a semi-simple ordered semigroup, then every ( $\in, \in \vee q_{k}$ )-fuzzy interior ideal is an ( $\in, \in \vee q_{k}$ )-fuzzy ideal of $S$.

Proof Let $F$ be an ( $\in, \in \vee q_{k}$ )-fuzzy interior ideal and $a, b \in S$. Then there exist $x, y, z \in S$

such that $a \leq x a y a z$. Then $F(a b) \geq F((x a y a z) b) \wedge \frac{1-k}{2}$ (because $F$ is an $\left(\epsilon, \in \vee q_{k}\right)$-fuzzy interior ideal)

$$
\begin{aligned}
& =F((x a y) a(z b)) \wedge \frac{1-k}{2} \\
& \geq\left(F(a) \wedge \frac{1-k}{2}\right) \wedge \frac{1-k}{2} \\
& =F(a) \wedge \frac{1-k}{2} .
\end{aligned}
$$

Similarly, we can show that $F(a b) \geq F(b) \wedge \frac{1-k}{2}$ for every $a, b \in S$. Thus $F$ is an ( $\in, \in \vee q_{k}$ )-fuzzy ideal of $S$.

From Propositions 3.10 and 3.14, we have the following Corollary:

Corollary 3.15 In semi-simple ordered semigroups, the concepts of an ( $\in, \in \vee q_{k}$ )-fuzzy interior and an ( $\in, \in \vee q_{k}$ )-fuzzy ideal coincide.

\section{Semiprime ( $\in, \in \vee q k$ )-fuzzy ideals}

In this section, we define semi-prime $\left(\in, \in \vee q_{k}\right)$-fuzzy ideals

in ordered semigroups, and prove that an ordered semigroup $S$ is left regular if and only if every ( $\in, \in \vee q_{k}$ )-fuzzy left ideal is semiprime.
Also, we prove that $S$ is intra-regular if and only if every $\left(\in, \in \vee q_{k}\right.$ )-fuzzy ideal is semiprime.

Definition 4.1 An ( $\in, \in \vee q_{k}$ )-fuzzy ideal $F$ of $S$ is called semiprime $\left(\epsilon, \in \vee q_{k}\right)$-fuzzy ideal of $S$ if

$(\forall x \in S)(\forall t \in(0,1])\left(\left[x^{2} ; t\right] \in F \Rightarrow[x ; t] \in \vee q_{k} F\right)$.

Theorem 4.2 An( $\in, \in \vee q_{k}$ )-fuzzy ideal $F$ of $S$ is a semiprime( $\in, \in \vee q_{k}$ )-fuzzy ideal of $S$ if and only if it satisfies the following condition:

$(\forall x \in S)\left(F(x) \geq F\left(x^{2}\right) \wedge \frac{1-k}{2}\right)$.

Proof Let $F$ be a semi-prime $\left(\in, \in \vee q_{k}\right)$-fuzzy ideal of $S$. On contrary, assume that there exists $x \in S$ such that $F(x)<F\left(x^{2}\right) \wedge \frac{1-k}{2}$. Choose $t \in(0,1]$ such that $F(x)<t \leq F\left(x^{2}\right) \wedge \frac{1-k}{2}$. Then $\left[x^{2} ; t\right] \in F$ but $F(x)<t$ and $F(x)+t+k<\frac{1-k}{2}+\frac{1-k}{2}+k=1$, and so $[x ; t] \in \vee \overline{q_{k}} F$, a contradiction. Therefore, $F(x) \geq F\left(x^{2}\right) \wedge \frac{1-k}{2}$ for all $x \in S$.

Conversely, let $\left[x^{2} ; t\right] \in F$ for some $t \in(0,1]$. Then $F\left(x^{2}\right) \geq t$ and we have, $F(x) \geq F\left(x^{2}\right) \wedge \frac{1-k}{2} \geq t \wedge \frac{1-k}{2}$. If $t>\frac{1-k}{2}$, then $F(x) \geq \frac{1-k}{2}$ and $F(x)+t+k>\frac{1-k}{2}+\frac{1-k}{2}+k=1$, it follows that $x ; t-{ }_{\mathrm{q}}{ }_{k} F$. If $t \leq \frac{1-k}{2}$, then $F(x) \geq t$ and $[x ; t] \in F$. Thus, $[x ; t] \in \vee \mathrm{q} k$, and consequently, $F$ is semiprime $\left(\in, \in \vee q_{k}\right)$-fuzzy ideal of $S$.

If we take $k=0$, in Theorem 4.2, we get the following corollary:

Corollary 4.3 An $(\in, \in \vee q)$-fuzzy ideal $F$ of $S$ is a semiprime $(\in, \in \vee q$ )-fuzzy ideal of $S$ if and only if it satisfies the following condition:

( $\forall x \in S)\left(F(x) \geq F\left(x^{2}\right) \wedge 0.5\right)$

Proof The proof follows from Theorem 4.2

Theorem 4.4 A fuzzy subset $F$ of $S$ is a semiprime $\left(\in, \in \vee q_{k}\right)$ fuzzy ideal of $S$ if and only if $U(F ; t)(\neq \phi)$ is a semiprime ideal of $S$, for all $t \in\left(0, \frac{1-k}{2}\right]$.

Proof Assume that $F$ is a semi-prime $\left(\in, \in \vee q_{k}\right)$-fuzzy ideal of $S$ . Let $x \in S$ such that $x^{2} \in U(F ; t)$, for some $t \in\left(0, \frac{1-k}{2}\right]$. Then $F\left(x^{2}\right) \geq t$ and by Theorem 4.2, we have $F(x) \geq F\left(x^{2}\right) \wedge \frac{1-k}{2} \geq t \wedge \frac{1-k}{2}=t$ and so $x \in U(F ; t)$. Thus, $U(F ; t)$ is semiprime ideal of $S$ Conversely, let $U(F ; t)$ is semiprime ideal of $S$, for all $t \in\left(0, \frac{1-k}{2}\right]$. If there exists $x \in S$ such that $F(x)<F\left(x^{2}\right) \wedge \frac{1-k}{2}$. Choose $t \in\left(0, \frac{1-k}{2}\right]$ such that $F(x)<t \leq F\left(x^{2}\right) \wedge \frac{1-k}{2}$, then $x^{2} \in U(F ; t)$ but $x \in \bar{x} U(F ; t)$, a contradiction. Thus, $F(x) \geq F\left(x^{2}\right) \wedge \frac{1-k}{2}$ for all $x \in S$. Therefore, $F$ is a semi-prime $\left(\in, \in \vee q_{k}\right)$-fuzzy ideal of $S$.

If we take $k=0$, in Theorem 4.4, we get the following corollary:

Corollary 4.5 A fuzzy subset $F$ of $S$ is a semiprime $(\in, \in \vee q)$ fuzzy ideal of $S$ if and only if $U(F ; t)(\neq \phi)$ is a semiprime ideal of $S$, for all $t \in(0,0.5]$.

Proof The proof follows from Theorem 4.4 .

Lemma 4.6 A non-empty subset $A$ of $S$ is a semiprime if and only if the characteristic function $\chi_{A}$ of $A$ is a semiprime( $\left.\in, \in \vee q_{k}\right)$ - 
fuzzy ideal of $S$.

Proof The proof is straightforward.

If we take $k=0$ in Lemma 4.6, then we have the following corollary:

Corollary 4.7 A non-empty subset $A$ of $S$ is a semiprime if and only if the characteristic function $\chi_{A}$ of $A$ is a semiprime $(\in, \in \vee$ q)-fuzzy ideal of $S$.

Lemma 4.8 (Khan and Shabir et al., 2009) Let $S$ be an ordered semigroup. Then the following statements are equivalent:

(1) $S$ is left (resp. right) regular.

(2) Every left ideal of $S$ is semiprime.

(3) $L(a)$ is semi-prime left ideal of $S$, for every $a \in S$.

(4) $L\left(a^{2}\right)$ is semi-prime left ideal of $S$, for every $a \in S$.

Lemma 4.9 (Khan and Shabir et al., 2009) Let $S$ be an ordered semigroup. Then the following statements are equivalent:

(1) $S$ is intra-regular.

(2) Every ideal of $S$ is semiprime.

(3) $I(a)$ is semi-prime ideal of $S$, for every $a \in S$.

(4) $I\left(a^{2}\right)$ is semi-prime left ideal of $S$, for every $a \in S$.

Lemma 4.10 An ordered semigroup $S$ is left regular if and only if every $\left(\in, \in \vee q_{k}\right)$-fuzzy left ideal of $S$ is semiprime.

Proof Let $S$ be left regular, $F$ an $\left(\in, \in \vee q_{k}\right)$-fuzzy left ideal of $S$ and $a \in S$. Since $S$ is left regular, there exists $x \in S$ such that $a \leq x a^{2}$. Then,

$F(a) \geq F\left(x a^{2}\right) \wedge \frac{1-k}{2} \geq\left(F\left(a^{2}\right) \wedge \frac{1-k}{2}\right) \wedge \frac{1-k}{2}=F\left(a^{2}\right) \wedge \frac{1-k}{2}$.

Thus, $F$ is semi-prime $\left(\in, \in \vee q_{k}\right)$-fuzzy left ideal of $S$.

Conversely, assume that every $\left(\in, \in \vee q_{k}\right)$-fuzzy left ideal $F$ of $S$ is semiprime. We consider the left ideal $L\left(a^{2}\right)$, generated by $a^{2}$. Then by Lemma 4.6 , the characteristic function $\chi_{L\left(a^{2}\right)}$ is an ( $\in, \in \vee q_{k}$ )-fuzzy semiprime ideal of $S$ and by hypothesis, $\chi_{L\left(a^{2}\right)}$ is a semiprime. Thus, by Lemma 4.6, $L\left(a^{2}\right)$ is semiprime left ideal of $S$ and by Lemma 4.8, $S$ is left regular.

In a similar way, we can prove that $S$ is right regular if and only if every ( $\in, \in \vee q_{k}$ )-fuzzy ideal of $S$ is semiprime $\left(\in, \in \vee q_{k}\right)$-fuzzy ideal of ${ }_{S}$ is semiprime.

Similarly, we have the following lemma:

Lemma 4.11 An ordered semigroup $S$ is intra-regular if and only if every ( $\in, \in \vee q_{k}$ )-fuzzy ideal of $S$ is semiprime.

If $(S, \cdot, \leq)$ is an ordered semigroup and $a \in S$, and $F$ a fuzzy subset of $S$, we denote by $I_{a}$ the subset of $S$ defined as follows:

$$
I_{a}:=\left\{b \in S \mid F(b) \geq F(a) \wedge \frac{1-k}{2}\right\} .
$$

Proposition 4.12 Let $S$ be an ordered semigroup and $F$ an ( $\in, \in \vee q_{k}$ )-fuzzy right ideal of $S$. Then $I_{a}$ is a right ideal of $S$ for every $a \in S$.

Proof Let $F$ be an ( $\in, \in \vee q_{k}$ )-fuzzy right ideal of $S$ and $a \in S$. Then $I_{a} \neq \phi$, because $a \in I_{a}$ for every $a \in S$. Let $b \in I_{a}$ and $x \in S$
. We have to prove that $b x \in I_{a}$. Since $F$ is an $\left(\in, \in \vee q_{k}\right)$-fuzzy right ideal of $S$, we have $F(b x) \geq F(b) \wedge \frac{1-k}{2}$. Since $b \in I_{a}$, we have

$$
F(b) \geq F(a) \wedge \frac{1-k}{2}
$$

$F(b) \wedge \frac{1-k}{2} \geq F(a) \wedge \frac{1-k}{2} \wedge \frac{1-k}{2}=F(a) \wedge \frac{1-k}{2}$.

Thus

$F(b x) \geq F(b) \wedge \frac{1-k}{2} \geq F(a) \wedge \frac{1-k}{2}$, hence $b x \in I_{a}$.

Let $b \in I_{a}$ and $S ? x \leq b$. Then $x \in I_{a}$. Indeed, since $F$ is an ( $\in, \in \vee q_{k}$ )-fuzzy right ideal of $S$, and $S ? x \leq b$, we have $F(x) \geq F(b) \wedge \frac{1-k}{2}$, and $x \in I_{a}$. Thus $I_{a}$ is a right ideal of $S$.

In a similar way we can prove that:

Proposition 4.13 Let $S$ be an ordered semigroup and $F$ an ( $\in, \in \vee q_{k}$ )-fuzzy left ideal of $S$. Then $I_{a}$ is a left ideal of $S$ for every $a \in S$.

Combining Propositions 4.12 and 4.13 we have the following:

Proposition 4.14 Let $S$ be an ordered semigroup and $F$ an ( $\in, \in \vee q_{k}$ )-fuzzy ideal of $S$.

Then $I_{a}$ is an ideal of $S$ for every $a \in S$.

\section{Upper and lower parts of ( $\in, \in \vee q k)$-fuzzy interior ideals}

Definition 5.1 Let $S$ be an ordered semigroup and $F$ a fuzzy subset of $S$.We define the upper part $F^{k+}$ and the lower part $F^{k-}$ of $F$ as follows:

$$
\begin{aligned}
& F^{k+}: S \rightarrow[0,1], x \mapsto F^{k+}(x)=F(x) \vee \frac{1-k}{2} \text { and } \\
& F^{k-}: S \rightarrow[0,1], x \mapsto F^{k-}(x)=F(x) \wedge \frac{1-k}{2} \text { for all } x \in S .
\end{aligned}
$$

Lemma 5.2 (Bhakat and Das, 1996) Let $F$ and $G$ be fuzzy subsets of an ordered semigroup. Then the following hold:

(1) $\left(F \wedge^{k} G\right)^{-}=\left(F^{-} \wedge^{k} G^{-}\right)$.

(2) $\left(F \vee^{k} G\right)^{-}=\left(F^{-} \vee^{k} G^{-}\right)$.

(3) $\left(F \circ^{k} G\right)^{-}=\left(F^{-} \circ^{k} G^{-}\right)$.

Definition 5.3 Let $A$ be a nonempty subset of an ordered semigroup. Then the upper part $F_{A}^{k+}$ and the lower part $F_{A}^{k-}$ of the characteristic function $F_{A}$ of $A$ are defined by:

and

$$
F_{A}^{k-}: S \rightarrow[0,1], x \mapsto F_{A}^{k-}(x):= \begin{cases}\frac{1-k}{2} \text { if } x \in A, \\ 0 \text { if } x \notin A,\end{cases}
$$

$$
F_{A}^{k+}: S \rightarrow[0,1], x \mapsto F_{A}^{k+}(x):= \begin{cases}1 & \text { if } x \in A, \\ \frac{1-k}{2} & \text { if } x \notin A .\end{cases}
$$

Lemma 5.4 (Bhakat and Das, 1996) If $A$ and $B$ be nonempty subsets of an ordered semigroup. Then the following hold:

(1) $\left(F_{A} \wedge^{k} F_{B}\right)^{-}=F_{A \cap B}^{k-}$.

(2) $\left(F_{A} \vee^{k} F_{B}\right)^{-}=F_{A \cup B}^{k-}$.

(3) $\left(F_{A} \circ^{k} F_{B}\right)^{-}=F_{(A B]}^{k-}$.

Lemma 5.5 The lower part of the characteristic function $F_{I}^{k-}$ is an $\left(\in, \in \vee q_{k}\right.$ )-fuzzy interior ideal of $S$ if and only if $I$ is an interior ideal of $S$.

Proof.The proof follows from Lemma 3.9. 
In the following we show that if $F$ is an $\left(\in, \in \vee q_{k}\right)$-fuzzy interior ideal of $S$ then $F^{k-}$ is a fuzzy interior ideal of $S$.

Proposition 5.6 Let $F$ be an $\left(\in, \in \vee q_{k}\right)$-fuzzy interior ideal of $S$, then $F^{k-}$ is a fuzzy interior ideal of $S$.

Proof.Let $F$ be an $\left(\in, \in \vee q_{k}\right)$-fuzzy interior ideal of $S$, then for all $x, y \in S$, we have $F(x y) \geq\left(F(x) \wedge F(y) \wedge \frac{1-k}{2}\right)$. Then $F(x y) \wedge \frac{1-k}{2} \geq\left(F(x) \wedge F(y) \wedge \frac{1-k}{2}\right)=\left(F(x) \wedge \frac{1-k}{2}\right) \wedge\left(F(y) \wedge \frac{1-k}{2}\right)$. Hence $\quad F^{k}{ }^{-}(x y) \geq\left(F^{k-}(x) \wedge F^{k-}(y)\right)$ and $F(x a y) \geq\left(F(a) \wedge \frac{1-k}{2}\right)$. Then $F(x a y) \wedge \frac{1-k}{2} \geq\left(F(a) \wedge \frac{1-k}{2}\right) \wedge \frac{1-k}{2}=F(a) \wedge \frac{1-k}{2}$. Hence $F^{k-}(x a y) \geq F^{k-}(a)$. Let $x, y \in S, x \leq y$, then by hypothesis, $F(x) \geq\left(F(y) \wedge \frac{1-k}{2}\right) \quad, \quad$ and $\quad$ we have $F(x) \wedge \frac{1-k}{2} \geq\left(F(y) \wedge \frac{1-k}{2}\right) \wedge \frac{1-k}{2}=F(y) \wedge \frac{1-k}{2}, \quad$ then $F(x) \wedge \frac{1-k}{2} \geq F(y) \wedge \frac{1-k}{2}$ and we have $F^{k-}(x) \geq F^{k-}(y)$. Thus $F^{k-}$ is a fuzzy interior ideal of $S$.

Lemma 5.7 The lower part of the characteristic function $F_{I}^{k-}$ is an ( $\in, \in \vee q_{k}$ )-fuzzy left ideal of $S$ if and only if $I$ is a left ideal of $S$ Similarly, we have the following Lemma:

Lemma 5.8 The lower part of the characteristic function $F_{I}^{k-}$ is an ( $\in, \in \vee q_{k}$ )-fuzzy right ideal of $S$ if and only if $I$ is a right ideal of $S$.

Combining Lemmas 5.7 and 5.8, we have the following:

Corollary 5.9 The lower part of the characteristic function $F_{I}^{k-}$ is an $\left(\in, \in \vee q_{k}\right)$-fuzzy ideal of $S$ if and only if $I$ is an ideal of $S$.

Definition 5.10 An ordered semigroup $S$ is called $\left(\in, \in \vee q_{k}\right)$-fuzzy left (resp. $\left(\in, \in \vee q_{k}\right)$-fuzzy right) simple if every $\left(\in, \in \vee q_{k}\right)$-fuzzy left (resp. ( $\in, \in \vee q_{k}$ )-fuzzy right) ideal is constant, that is, for every $a, b \in S$, we have, $F^{k-}(a)=F^{k-}(b)$, for every $\left(\in, \in \vee q_{k}\right)$-fuzzy left (right) ideal $F$ of $S$. An ordered semigroup $S$ is called ( $\left.\in, \in \vee q_{k}\right)$-fuzzy simple if it is both $\left(\in, \in \vee q_{k}\right)$-fuzzy left and right simple.

Theorem 5.11 An ordered semigroup $(S,, \leq)$ is simple if and only if it is $\left(\in, \in \vee q_{k}\right)$-fuzzy simple.

Proof. $\boldsymbol{\nearrow}$. Let $F$ be an $\left(\in, \in \vee q_{k}\right)$-fuzzy ideal of $S$ and $a, b \in S$ . Using Proposition 4.14, we get, $I_{a}$ is an ideal of $S$. Since $S$ is simple, it follows that $I_{a}=S$ so that $b \in I_{a}$. Thus $F(b) \geq F(a) \wedge \frac{1-k}{2}$, and so

$$
F^{k-}(b)=F(b) \wedge \frac{1-k}{2} \geq F(a) \wedge \frac{1-k}{2}=F^{k-}(a) .
$$

Similarly, we get $F^{k-}(b) \leq F^{k-}(a)$. Hence $F^{k-}(a)=F^{k-}(b)$, and thus $S$ is $\left(\in, \in \vee q_{k}\right)$-fuzzy simple.

$\Leftarrow$. Suppose that $S$ contains proper ideals and let $I$ be an ideal of $S$ such that $I \neq S$. Using Corollary 5.9, $F_{I}^{k-}$ is an $\left(\in, \in \vee q_{k}\right)$ fuzzy ideal of $S$. Let $x \in S$. Since $S$ is $\left(\in, \in \vee q_{k}\right)$-fuzzy simple, $F_{I}^{k-}$ is a constant function, that is, $F_{I}^{k-}(x)=F_{I}^{k-}(b)$ for every $b \in S$. Thus, for any $a \in I$, we have $F_{I}^{k-}(x)=F_{I}^{k-}(a)=\frac{1-k}{2}$ and so $x \in I$. Therefore $S=I$, a contradiction. Consequently, $S$ is simple.

Lemma 5.12 (Shabir and Khan, 2009). An ordered semigroup $S$ is simple if and only if for every $a \in S$, we have $S=(S a S]$.

Theorem 5.13 An ordered semigroup $(S, \cdot, \leq)$ is simple if and only if for every ( $\in, \in \vee q_{k}$ )-fuzzy ideal $F$ of $S$, we have $F^{k-}(a)=F^{k-}(b)$ for every $a, b \in S$.

Proof. $\boldsymbol{\lambda}$. Suppose that $F$ is an $\left(\in, \in \vee q_{k}\right)$-fuzzy interior ideal of $S$ and $a, b \in S$. Since $S$ is simple and $b \in S$, by Lemma 5.12, we have $S=(S a S]$. Since $a \in S$, we have $a \in(S b S]$. Then $a \leq x b y$ for some $x, y \in S$. Since $F$ is an $\left(\in, \in \vee q_{k}\right)$-fuzzy interior ideal of $S$, we have

$$
\begin{aligned}
& \begin{aligned}
F(a) & \geq F(x b y) \wedge \frac{1-k}{2} \\
& =F(x(b y)) \wedge \frac{1-k}{2} \\
& \geq\left(F(b) \wedge \frac{1-k}{2}\right) \wedge \frac{1-k}{2}\left(\text { since } F \text { is an }\left(\epsilon, \in \vee q_{k}\right) \text { - fuzzy interior ideal }\right) \\
& =F(b) \wedge \frac{1-k}{2},
\end{aligned} \\
& \text { hence } F(a) \geq F(b) \wedge \frac{1-k}{2} \text { and } \\
& F(a) \wedge \frac{1-k}{2} \geq\left(F(b) \wedge \frac{1-k}{2}\right) \wedge \frac{1-k}{2}=F(b) \wedge \frac{1-k}{2} \text {. Thus } \\
& F^{k-}(a) \geq F^{k-}(b) \cdot \text { By a similar way, we can find that } \\
& F^{k-}(b) \geq F^{k-}(a) . \text { Therefore } F^{k-}(a)=F^{k-}(b) \text { for every } a, b \in S
\end{aligned}
$$

Assume that $F$ is an $\left(\in, \in \vee q_{k}\right.$ )-fuzzy interior ideal of $S$ , then by Proposition 3.14, $F$ is an $\left(\in, \in \vee q_{k}\right)$-fuzzy ideal of $S$. By hypothesis, $F^{k-}(a)=F^{k-}(b)$ for every $a, b \in S$. Thus $S$ is an $\left(\in, \in \vee q_{k}\right)$-fuzzy simple and by Theorem 5.11, $S$ is simple.

Theorem 5.14 An ordered semigroup $S$ is intra-regular if and only if for every $\left(\in, \in \vee q_{k}\right)$-fuzzy ideal $F$ of $S$, we have $F^{k-}(a)=F^{k-}\left(a^{2}\right)$ for all $a \in S$.

Proof. $\boldsymbol{\nearrow}$. Let $F$ be an $\left(\in, \in \vee q_{k}\right)$-fuzzy ideal of $S$ and let $a \in S$. Then there exist $x, y \in S$ such that $a \leq x a^{2} y$ and

$$
\begin{aligned}
F(a) & \geq F\left(x a^{2} y\right) \wedge \frac{1-k}{2} \\
& =F\left(x\left(a^{2} y\right)\right) \wedge \frac{1-k}{2} \\
& \geq\left(F\left(a^{2} y\right) \wedge \frac{1-k}{2}\right) \wedge \frac{1-k}{2} \\
& =F\left(a^{2} y\right) \wedge \frac{1-k}{2} \geq\left(F\left(a^{2}\right) \wedge \frac{1-k}{2}\right) \wedge \frac{1-k}{2} \\
& =F\left(a^{2}\right) \wedge \frac{1-k}{2}=F^{k-}\left(a^{2}\right)
\end{aligned}
$$

On the other hand,

$F^{k-}\left(a^{2}\right)=F\left(a^{2}\right) \wedge \frac{1-k}{2} \geq\left(F(a) \wedge \frac{1-k}{2}\right) \wedge \frac{1-k}{2}=F(a) \wedge \frac{1-k}{2}=F^{k-}(a)$. Hence

$F^{k-}(a)=F^{k-}\left(a^{2}\right) \cdot$

Conversely, consider the ideal $I\left(a^{2}\right)=\left(a^{2} \cup S a^{2} \cup a^{2} S \cup S a^{2} S\right]$, generated by $a^{2}$. Then by Corollary 5.9, $F_{I\left(a^{2}\right)}^{k-}$ is an $\left(\in, \in \vee q_{k}\right)$ - 
fuzzy ideal of $S$ and by hypothesis $F_{I\left(a^{2}\right)}^{\prime \prime}(a)=F_{I\left(a^{2}\right)}^{\lambda}\left(a^{2}\right)=\frac{I-k}{2}$, hence $a \in I\left(a^{2}\right)=\left(a^{2} \cup S a^{2} \cup a^{2} S \cup S a^{2} S\right]$. Thus $a \leq a^{2}$ or $a \leq x a^{2}$ or $a \leq a^{2} x$ or $a \leq x a^{2} y \quad$ for some $\quad x, y \in S$. If $a \leq a^{2}=a a \leq a^{2} a^{2}=a a^{2} a \in S a^{2} S$ and $a \in\left(S a^{2} S\right]$. Similarly, for other cases we have $a \leq u a^{2} v$ for some $u, v \in S$. Thus, $S$ is intraregular.

Theorem 5.15 Let $S$ be an intra-regular ordered semigroup and $F$ is an $\left(\in, \in \vee q_{k}\right)$-fuzzy ideal of $S$. Then for every $a, b \in S$, we have

$F^{k-}(a b)=F^{k-}(b a)$.

Proof Let $a, b \in S$. Then by Theorem 5.14, we have

$$
\begin{aligned}
F^{k-}(a b) & =F^{k-}\left((a b)^{2}\right)=F^{k-}((a b)(a b))=F^{k-}(a(b a) b) \\
& \geq F^{k-}(b a) .
\end{aligned}
$$

By symmetry we have $\quad F^{k-}(b a) \geq F^{k-}(a b)$. Thus $F^{k-}(a b)=F^{k-}(b a)$ for every $a, b \in S$.

In semi-simple ordered semigroups are characterized by the properties of their ( $\in, \in \vee q$ )-fuzzy interior ideals (Shabir and Khan, 2014). Next, we are characterizing the semi-simple ordered semigroups by the properties of lower parts of $\left(\in, \in \vee q_{k}\right)$-fuzzy interior ideals.

Proposition 5.16 If $S$ is semi-simple ordered semigroup and $F$, $G$ are $\left(\in, \in \vee q_{k}\right)$-fuzzy interior ideals. Then $\left(F \circ^{k} G\right)^{-} \leq\left(F \wedge^{k} G\right)^{-}$

Proof Let $S$ be a semi-simple and $F$ an $\left(\in, \in \vee q_{k}\right)$-fuzzy interior ideal of $S$. If $A_{a}=\phi$ then $\left(F \circ^{k} G\right)^{-}(a)=(F \circ G)(a) \wedge \frac{1-k}{2}=0 \wedge \frac{1-k}{2}=0 \leq(F \wedge G)(a) \wedge \frac{1-k}{2}=\left(F \wedge^{k} G\right)^{-}(a)$. Let $A_{a} \neq \phi$, then

$$
\begin{aligned}
\left(F \circ^{k} G\right)^{-}(a) & =(F \circ G)(a) \wedge \frac{1-k}{2} \\
& =\left[\bigvee_{(y, z) \in A_{a}}(F(y) \wedge G(z))\right] \wedge \frac{1-k}{2} \\
& =\bigvee_{(y, z) \in A_{a}}\left(F(y) \wedge G(z) \wedge \frac{1-k}{2}\right) \\
& =\bigvee_{(y, z) \in A_{u}}\left(\left(F(y) \wedge \frac{1-k}{2}\right) \wedge\left(G(z) \wedge \frac{1-k}{2}\right) \wedge \frac{1-k}{2}\right) .
\end{aligned}
$$

Since $S$ is semi-simple and $F$ and $G$ are $\left(\in, \in \vee q_{k}\right)$-fuzzy interior ideals, so by Proposition 3.14, $F$ and $G$ are $\left(\in, \in \vee q_{k}\right.$ )-fuzzy ideal of $S$. Since $a \leq y z$, we have $F(a) \geq F(y z) \wedge \frac{1-k}{2} \geq F(y) \wedge \frac{1-k}{2} \quad$ and

$G(a) \geq G(y z) \wedge \frac{1-k}{2} \geq G(z) \wedge \frac{1-k}{2}$.

Thus

$$
\begin{aligned}
& \underset{(y, z) A_{a}}{\vee}\left(\left(F(y) \wedge \frac{1-k}{2}\right) \wedge\left(G(z) \wedge \frac{1-k}{2}\right) \wedge \frac{1-k}{2}\right) \\
\leq & \vee(y, z) \in A_{a} \\
= & \bigvee_{(y, z) \in A_{a}}\left(F(a) \wedge G(a) \wedge \frac{1-k}{2}\right) \\
= & (F \wedge G)(a) \wedge \frac{1-k}{2}=\left(F \wedge^{k} G\right)^{-}(a) .
\end{aligned}
$$

Therefore $\left(F \circ^{k} G\right)^{-}(a) \leq\left(F \wedge^{k} G\right)^{-}(a)$.

Remark 5.17 If $S$ is a semi-simple and $F$ an ( $\left.\in, \in \vee q_{k}\right)$-fuzzy interior ideal of $S$. Then $\left(F \circ^{k} F\right)^{-} \leq F^{k-}$
Theorem 5.18 An ordered semigroup $S$ is semi-simple if and only if for every ( $\in, \in \vee q_{k}$ )-fuzzy interior ideal $F$ and $G$ of $S$, we have $\left(F \circ^{k} G\right)^{-}=\left(F \wedge^{k} G\right)^{-}$.

Proof Let $S$ be a semi-simple ordered semigroup and $F, G$ are ( $\in, \in \vee q_{k}$ )-fuzzy interior ideals of $S$. Since $S$ is semi-simple so for each $a \in S$, there exist $\quad x, y, z \in S$ such that $a \leq \operatorname{axayaz} \leq \operatorname{ax}($ axayaz $) y a z=((a x) a(x a y)(a z y) a(z))$, then $((a x) a(x a y),(a z y) a(z)) \in A_{a}$ and $A_{a} \neq \phi$. Thus

$$
\begin{aligned}
\left(F \circ^{k} G\right)^{-}(a) & =\left(F \circ^{k} G\right)(a) \wedge \frac{1-k}{2} \\
& =\left[\bigvee_{(y, z) \in A_{a}}(F(y) \wedge G(z))\right] \wedge \frac{1-k}{2} \\
& =\bigvee_{(y, z) \in A_{a}}\left(F(y) \wedge G(z) \wedge \frac{1-k}{2}\right) \\
& \geq\left(F((a x) a(x a y)) \wedge G((a z y) a(z)) \wedge \frac{1-k}{2}\right) \\
& \geq\left(F(a) \wedge \frac{1-k}{2}\right) \wedge\left(G(a) \wedge \frac{1-k}{2}\right) \wedge \frac{1-k}{2} \\
& =\left(F(a) \wedge G(a) \wedge \frac{1-k}{2}\right)=(F \wedge G)(a) \wedge \frac{1-k}{2} \\
& =\left(F \wedge^{k} G\right)^{-}(a) .
\end{aligned}
$$

This implies that $\left(F \wedge^{k} G\right)^{-} \leq\left(F \circ^{k} G\right)^{-}$. On the other hand, by Proposition 5.16, we have $\left(F \circ^{k} G\right)^{-} \leq\left(F \wedge^{k} G\right)^{-}$and so $\left(F \circ \circ^{k} G\right)^{-}=\left(F \wedge^{k} G\right)^{-}$.

Conversely, assume that $\left(F \circ^{k} G\right)^{-}=\left(F \wedge^{k} G\right)^{-}$for every $\left(\in, \in \vee q_{k}\right.$ )-fuzzy interior ideal $F$ and $G$ of $S$. Let $I$ be an interior ideal of $S$, then $I$ is an ideal of $S$ and by Lemma 5.5, the characteristic function $F_{I}^{k-}$ of $I$ is an $\left(\in, \in \vee q_{k}\right.$ )-fuzzy interior ideal of $S$. By hypothesis and Lemma 5.4 (3), we have

$$
F_{I}^{k-}=F_{I}^{k-} \wedge F_{I}^{k-}=\left(F_{I} \wedge^{k} F_{I}\right)^{-}=\left(F_{I} o^{k} F_{I}\right)^{-}=F_{\left(I^{2}\right]}^{k-}
$$

Using Lemma 5.4 again we have $I=\left(I^{2}\right]$. Thus $S$ is semi-simple.

By Remark 5.17 and Theorem 5.18, we have the following theorem:

Theorem 5.19 An ordered semigroup $S$ is semi-simple if and only if for every $\left(\in, \in \vee q_{k}\right)$-fuzzy interior ideal $F$ of $S$, we have $\left(F \circ^{k} F\right)^{-}=F^{k-}$. 


\section{References}

[1] Ahsan, J., Li, K.Y., Shabir, M., (2002). Semigroups characterized by their fuzzy bi-ideals. Journal of Fuzzy Mathematics, 10 (2): 441-449.

[2] Bhakat, S.K., (1999). $(\in \vee q)$-level subset. Fuzzy Sets and Systems, 103(3):529-533.

[3] Bhakat, S.K., Das, P., (1992). On the definition of a fuzzy subgroup. Fuzzy Sets and Systems, 51(2):17-21.

[4] Bhakat, S.K., Das, P., (1996a). $(\in, \in \vee q)$-fuzzy subgroups. Fuzzy Sets and Systems, 80(3), 359-368.

[5] Bhakat, S.K., Das, P., (1996b). Fuzzy subrings and ideals redefined. Fuzzy Sets and Systems, 81(3): 383-393.

[6] Das, P.S., (1981). Fuzzy groups and level subgroups. Journal of Mathematical Analysis and Applications, 84(1): 264-269.

[7] Davvaz, B., (2006). $(\in, \in \vee q)$-fuzzy sub earrings and ideals. Soft Computing, 10(3):206-2011.

[8] Davvaz, B., Corsini, P., (2007). Redefined fuzzy $H_{v}$. submodules and many valued implications. Information Science, 177(3): 865-875.

[9] Davvaz, B., Dudek, W.A., Jun, (2006). Intuitionistic fuzzy $H_{v}$ -submodules. Information Science, 176: 285-300.

[10] Davvaz, B., Khan, A., (2011). Characterizations of regular ordered semigroups in terms of $(\alpha, \beta)$-fuzzy generalized bi-ideals. Information Science,181 (9): 1759-1770.

[11] Davvaz, B., Mahdavipour, M., (2006). Roughness in modules. Information Science, 176 (24): 3658-3674.

[12] Jun, Y.B., Khan, A., Shabir, M., (2009). Ordered semigroups characterized by their $(\in, \in \vee q)$-fuzzy bi-ideals. Bulletin of the Malaysian Mathematical Sciences Society, 32(3): 391-408.

[13] Jun, Y.B., Khan, A., Sarmin, N.H., Khan, F.M., (2012). Ordered semigroups characterized by $\left(\in, \in \vee q_{k}\right.$ )-fuzzy generalized bi-ideals. Neural Computing and Applications, 21(1): 121-132.

[14] Jun, Y.B., Song, S.Z., (2006). Generalized fuzzy interior ideals in semigroups. Information Sciences, 176(20): 3079-3093.

[15] Jun, Y.B., Xu, Y., Ma, J., (2007). Redefined fuzzy implicative filters. Information Science, 177(6): 1422-1429.

[16] Kazanci, O., Yamak, S., Generalized fuzzy bi-ideals of semigroup. Soft Computing, DOI 10.1007/s00500-008-0280-5.

[17] Kehayopulu, N., (1992). On left regular and left duo poesemigroups. Semigroup Forum, 44(3): 306-313.

[18] Kehayopulu, N., (1993). On intra-regular ordered semigroups. Semigroup Forum, 46(1): 271-278.

[19] Khan, A., Shabir, M., (2009). ( $\alpha, \beta$ )-fuzzy interior ideals in ordered semigroups. Lobachevskii Journal of Mathematics, 30(1): 30-39.

[20] Khan, A., Jun, Y.B., Abbas, Z., (2012). Characterizations of ordered semigroups by $(\in, \in \vee q)$-fuzzy interior ideals. Neural Computing and Applications, DOI: 10.1007/s00521-010-0463-8.
[21] Khan, A., Sarmin, N.H., Davvaz, B., Khan, H.N., (2012). New types of fuzzy bi-ideals in ordered semigroups. Neural Computing and Applications, 21(1): 295-305.

[22] Kuroki, N., (1979). Fuzzy bi-ideals in semigroups. Comment. Math. Univ. St. Pauli XXVIII-1:

[23] Ma, X., Zhan, J., Xu, Y., (2007). Generalized fuzzy filters of $R_{0}$-algebras. Soft Computing 11: 1079-1087.

[24] Mordeson, J.N., Malik, D.S., and Kuroki, N., (2003). Fuzzy Semigroups, Studies in Fuzziness and Soft Computing. SpringerVerlag Berlin, 131.

[25] Murali, V., (2004). Fuzzy points of equivalent fuzzy subsets, Inform. Science Citation Index, 158: 277-288.

[26] Pu, P.M., Liu, Y.M., (1980). Fuzzy topology I, neighborhood structure of a fuzzy point and Moore-Smith convergence. Journal of Mathematical Analysis and Applications, 76(2): 571-599.

[27] Shabir, M., Jun, Y.B., Nawaz, Y., (2010). Characterizations of regular semigroups by $(\alpha, \beta)$-fuzzy ideals. Computers \& Mathematics with Applications, 59: 161-175.

[28] Shabir, M., Jun, Y.B., Nawaz, Y., (2010). Semigroups characterized by $\left(\in, \in \vee q_{k}\right)$-fuzzy ideals. Computers \& Mathematics with Applications, 60(5): 1473-1493.

[29] Shabir, M., Khan, A., (2008). Characterizations of ordered semigroups by the properties of their fuzzy generalized bi-ideals. New Mathematics and Natural Computation, 4 (2): 237-250.

[30] Shabir, M., Khan, A., (2009). Intuitionistic fuzzy interior ideals in ordered semigroups. Journal of Applied Mathematics \& Informatics, 27 (5-6): 1447-1457.

[31] Shabir, M., Khan, A., (2014). On fuzzy ordered semigroups. Information Sciences, 274: 236-248.

[32] Zadeh, L.A., (1965). Fuzzy Sets. Information and control, 8(3): $338-353$.

[33] Zhan, J., Davvaz, B., Shum, K.P., (2008). A new view of fuzzy hyper near-rings. Information Science, 178(2): 425-438. 